\title{
Generalized IIB supergravity from exceptional field theory
}

\author{
Arnaud Baguet, Marc Magro and Henning Samtleben \\ Laboratoire de Physique, Université Claude Bernard Lyon 1, Ens de Lyon, CNRS, \\ F-69342 Lyon, France \\ E-mail: arnaud.baguet@ens-lyon.fr, marc.magro@ens-lyon.fr, \\ henning.samtleben@ens-lyon.fr
}

ABSTRACT: The background underlying the $\eta$-deformed $A d S_{5} \times S^{5}$ sigma-model is known to satisfy a generalization of the IIB supergravity equations. Their solutions are related by T-duality to solutions of type IIA supergravity with non-isometric linear dilaton. We show how the generalized IIB supergravity equations can be naturally obtained from exceptional field theory. Within this manifestly duality covariant formulation of maximal supergravity, the generalized IIB supergravity equations emerge upon imposing on the fields a simple Scherk-Schwarz ansatz which respects the section constraint.

KeYwords: String Duality, Supergravity Models, Integrable Field Theories

ArXiv EPRINT: 1612.07210 


\section{Contents}

1 Introduction 1

2 Generalized IIB supergravity $\quad 4$

2.1 Generalized field equations and Bianchi identities 4

2.2 Solution of the Bianchi identities 5

$\begin{array}{lll}3 & \text { Exceptional field theory } & 7\end{array}$

$\begin{array}{lll}3.1 & \mathrm{E}_{6(6)} \text { exceptional field theory } & 7\end{array}$

3.2 Section constraints and IIA/IIB/generalized supergravity 8

$\begin{array}{llr}3.3 & \text { Scherk-Schwarz ansatz } & 9\end{array}$

3.4 Induced deformation 11

4 Conclusions $\quad 14$

\section{Introduction}

Integrability plays a key role in the study of AdS/CFT correspondence [1-3] between Type IIB superstring theory on the $A d S_{5} \times S^{5}$ background and the maximally supersymmetric Yang-Mills gauge theory in four dimensions (see [4] for a review). The former superstring theory is described by the Metsaev-Tseytlin action [5]. This action is the sum of two terms: a kinetic one and an exact Wess-Zumino-like one. The value of the coefficient in front of the Wess-Zumino term is fixed by requiring invariance under $\kappa$-symmetry. There is a very nice interplay between $\kappa$-symmetry and integrability. Indeed, the value of the aforementioned coefficient which guaranties $\kappa$-symmetry also ensures integrability. This means that the equations of motion admit a zero-curvature formulation in terms of a Lax pair [6] (see [7] for a review).

Recall that $\kappa$-symmetry is a fermionic gauge invariance. It has been shown in [8-10] that it is possible to deform the $A d S_{5} \times S^{5}$ superstring while maintaining both properties of integrability and invariance under a local fermionic symmetry. The $\sigma$-model constructed in [8] has been dubbed $\eta$-deformation. The one constructed in [9] is sometimes also designated as $\eta$-deformation in the literature. These theories are also called inhomogeneous/homogeneous Yang-Baxter deformations because their definitions involve a $R$-matrix, which is a solution of an inhomogeneous/homogeneous equation respectively. The latter equations are the modified classical Yang-Baxter equation and the classical Yang-Baxter equation respectively. The deformation proposed in [10] has been called $\lambda$ deformation of the $A d S_{5} \times S^{5}$ superstring. The actions which have been put forward in $[8,9]$ are deformations of the Metsaev-Tseytlin action while the action associated with the $\lambda$ deformation [10] appears as a deformation of the non-abelian T-dual of the $A d S_{5} \times S^{5}$ 
superstring $\sigma$-model. Inhomogeneous Yang-Baxter deformations are related [11-14] by Poisson-Lie T-duality $[15,16]$ and analytic continuation to $\lambda$-deformations. Such deformations generalise techniques applied to the principal chiral model and symmetric space $\sigma$-models [17-21]. These deformations, some further generalisation as well as lower dimensional cases have been thoroughly explored in many articles (see references for instance in the thesis [22] and the reviews [23, 24]).

It has been shown recently that the actions of the $\eta$ - and $\lambda$-deformations can be put on the Green-Schwarz form and that their fermionic local symmetry corresponds to standard $\kappa$-symmetry [25]. On a more general and fundamental level, a full understanding of these models from a supergravity perspective has required to revisit and underpin precisely the relationship between $\kappa$-symmetry and supergravity. This has been achieved a short while ago [26] and may be summarised as follows. Conditions to have classical $\kappa$-symmetry of Green-Schwarz $\sigma$-model are not equivalent ${ }^{1}$ to Type IIB supergravity equations but to the generalisation of these equations which has been derived in [26, 28]. An equivalent statement is that classical $\kappa$-symmetry does not imply two-dimensional Weyl invariance for the $\sigma$-model but only scale invariance.

The generalized supergravity equations comprise a Killing vector field $K$ and ordinary Type IIB supergravity equations are recovered whenever $K$ vanishes. For instance, the background associated with the $\lambda$-deformation has no isometry. This explains why the $\lambda$-deformation defines generically [25] a Type IIB supergravity background (see also [29-31] and [32, 33]). On the contrary, the Arutyunov-Borsato-Frolov (ABF) background $[22,34,35]$, which corresponds to the $\eta$-deformation in [8], is a solution of the generalized supergravity equations [28]. A general discussion, including the case of homogeneous Yang-Baxter deformations, and examples of the latter may be found in [25] and in [36-39] respectively.

The presence of a Killing vector field plays an important role. Indeed, solutions of generalized Type II supergravity are related by formal T-duality to ordinary supergravity solutions. This may be illustrated by two examples [28]. Consider firstly the Hoare-Tseytlin (HT) Type IIB background described in [40]. It consists of a metric $\widetilde{G}$, a (imaginary) 5form $\widetilde{F}_{5}$ and a dilaton $\widetilde{\phi}$. The metric and the product $\widetilde{\mathcal{F}}_{5}=e^{\widetilde{\phi}} \widetilde{F}_{5}$ are invariant under a $\mathrm{U}(1)^{6}$ isometry. However, the dilaton contains a linear non-isometric term, which breaks four of these isometries. Applying a formal T-duality in all isometric directions on $\widetilde{G}$ and $\widetilde{\mathcal{F}}_{5}$ leads [40] to the ABF background. The second example is simpler and explains the mechanism at work. We reproduce it from [28]. Consider the equations

$$
\widetilde{R}_{m n}+2 \widetilde{\nabla}_{m} \widetilde{\nabla}_{n} \widetilde{\phi}=0, \quad \text { and } \quad \widetilde{R}+4 \widetilde{\nabla}^{2} \widetilde{\phi}-4 \partial_{m} \widetilde{\phi} \partial^{m} \widetilde{\phi}=0,
$$

for a metric and dilaton of the form

$$
\widetilde{d s}^{2}=e^{2 a(x)}\left[d \widetilde{y}+A_{\mu}(x) d x^{\mu}\right]^{2}+g_{\mu \nu}(x) d x^{\mu} d x^{\nu} \quad \text { and } \quad \widetilde{\phi}(\widetilde{y}, x)=-c \widetilde{y}+f(x),
$$

with $c$ a constant. The metric has an isometry which is broken by the linear term in the dilaton. Applying T-duality to the metric leads to the metric and B-field:

$$
d s^{2}=e^{-2 a(x)} d y^{2}+g_{\mu \nu}(x) d x^{\mu} d x^{\nu} \quad \text { and } \quad B=A_{\mu}(x) d y \wedge d x^{\mu} .
$$

\footnotetext{
${ }^{1}$ See also [27] for a related discussion in the pure spinor formulation.
} 
Introduce then the vector $X=K+Z$, where the non-zero components of the Killing vector $K$ and $Z$ are $K_{y}=c e^{-2 a(x)}$ and $Z_{\mu}=\partial_{\mu}(f-a)-B_{\mu y} K^{y}$. Then, equations (1.1) become for $X$ and the metric and the field strength $H$ of the B-field in (1.2)

$$
R_{m n}+\nabla_{m} X_{n}+\nabla_{n} X_{m}=0, \quad \text { and } \quad R-\frac{1}{12} H_{m n p}^{2}+4 \nabla_{m} X^{m}-4 X_{m} X^{m}=0 .
$$

When $c$ vanishes, $K$ vanishes as well. Then $X_{m}=Z_{m}=\partial_{m}(f-a) \equiv \partial_{m} \phi$ and therefore equations (1.3) generalise equations (1.1).

The generalized IIB equations from [28] may be obtained by applying an analogous generalized T-duality to the full field equations of IIA supergravity again with non-isometric linear dilaton. This suggests that they should have a natural place in the manifestly duality covariant formulation of maximal supergravities - the so-called exceptional field theories (ExFT) [41-43]. These give a U-duality covariant unified description of IIA and IIB supergravity on a formally enhanced exceptional space-time. According to the solution of the section constraint which selects the physical coordinates, their field equations reproduce the standard IIA and the IIB field equations, respectively. We will show in this article, that also the generalized IIB supergravity equations are straightforwardly obtained from exceptional field theory upon choosing a particular solution of the section constraint.

Our framework for this article is the $\mathrm{E}_{6(6)}$ covariant exceptional field theory from [41, 42], most adapted to the $5+5$ split of the IIB coordinates for the undeformed $A d S_{5} \times S^{5}$ background. In $\mathrm{E}_{6(6)}$ ExFT, the $A d S_{5}$ coordinates $\left\{x^{\mu}\right\}$ remain external coordinates while the 5 coordinates $\left\{y^{a}\right\}$ describing the $S^{5}$-geometry of the undeformed background, are internal and formally embedded into 27 coordinates $\left\{Y^{M}\right\}$ transforming in the fundamental representation of the group $\mathrm{E}_{6(6)}$. Dependence of all fields on the 27 coordinates is heavily constrained by the section constraint stating that

$$
d^{K M N} \partial_{M} \otimes \partial_{N} \equiv 0,
$$

whenever the derivatives act on the various fields of the theory. Here, $d^{K M N}$ denotes the cubic invariant tensor of $\mathrm{E}_{6(6)}$. The field content of this ExFT is given in terms of $\mathrm{E}_{6(6)}$ covariant fields, most notably a group-valued symmetric $27 \times 27$ matrix $\mathcal{M}_{M N}$ parametrizing the coset space $\mathrm{E}_{6(6)} / \mathrm{USp}(8)$. The type IIB theory is recovered upon breaking $\mathrm{E}_{6(6)}$ down to its subgroup $\mathrm{SL}(5) \times \mathrm{SL}(2) \times \mathrm{GL}(1)_{\mathrm{IIB}}$, such that

$$
\begin{aligned}
\mathbf{2 7} & \longrightarrow(5,1)_{+4}+\left(5^{\prime}, 2\right)_{+1}+(10,1)_{-2}+(1,2)_{-5}, \\
\left\{Y^{M}\right\} & \longrightarrow\left\{y^{a}, \tilde{y}_{a \alpha}, \tilde{y}^{a b}, \tilde{y}_{\alpha}\right\}, \quad a=1, \ldots, 5, \quad \alpha= \pm .
\end{aligned}
$$

Restricting the dependence of all fields to the 5 coordinates $\left\{y^{a}\right\}$ of highest grading under the GL(1) IIB provides a solution to (1.4). Decomposing the matrix $\mathcal{M}_{M N}$ into its various blocks according to (1.5) allows to identify the explicit dictionary to the various components of the IB fields [44]. The field equations of ExFT in this case reproduce the equations of standard IIB supergravity.

We will show in this article that generalized IIB supergravity equations are obtained upon choosing a different solution of the section condition. Recall that among the ingredients of the generalized equations features a Killing vector field $K^{a}$ which after splitting 
$\left\{y^{a}\right\}=\left\{y^{i}, y^{*}\right\},(i=1, \ldots, 4)$, we choose along the fixed direction $K^{a}=\delta_{*}^{a}$, such that $\partial_{*} \Phi=0$ for all fields of the theory. In this case, the section constraint (1.4) admits a different (and inequivalent) solution in which we restrict the dependence of all fields to the subset $\left\{y^{i}, \tilde{y}_{*+}\right\}$, i.e. allow for an additional dependence on the coordinate $\tilde{y}_{*+}$. We will show that upon imposing a particular dependence on $\tilde{y}_{*+}$ (in terms of a generalized Scherk-Schwarz reduction ansatz), the field equations of exceptional field theory precisely reproduce the generalized IIB supergravity equations.

We should stress that the existence of an inequivalent solution to the section constraint does not come as a surprise but this choice of coordinates is equivalent (after rotation) to selecting the IIA coordinates $\tilde{y}_{a+}$ in (1.5), in which case ExFT reproduces the standard IIA theory. This precisely amounts to the fact that generalized IIB supergravity can be obtained via T-duality from a sector of IIA supergravity. Since the framework of exceptional field theory is manifestly duality covariant, we do not have to go through this duality explicitly but can simply absorb its effect into a rotation of the extended coordinates. Evaluating ExFT according to the IIB dictionary however in the new coordinates $\left\{y^{i}, \tilde{y}_{*+}\right\}$ with proper Scherk-Schwarz ansatz in $\tilde{y}_{*+}$ then directly yields the generalized IIB supergravity equations.

The rest of the article is organized as follows: in section 2 we collect the generalized IIB supergravity equations. In section 3 we start with a brief review of the relevant $\mathrm{E}_{6(6)}$ exceptional field theory and present the generalized Scherk-Schwarz ansatz that governs the $\tilde{y}_{*+}$-dependence of the fields. We work out the ExFT field equations with this ansatz and show that they yield the generalized IIB supergravity equations.

While we were writing up these results, Sakatani, Uehara, and Yoshida submitted the interesting paper [45] to the archive which in a similar spirit relates the NS-NS truncation of the generalized type IIB supergravity equations to the $O(d, d)$ covariant double field theory [46-49].

\section{Generalized IIB supergravity}

\subsection{Generalized field equations and Bianchi identities}

We recall in this section the bosonic generalized IIB supergravity equations which have been derived in [28]. Their fermionic completion has been found in [26]. The equations are expressed in string frame. The equations for the metric $G_{m n}$ and the $B$-field $B_{m n+}$ are

$$
\begin{aligned}
R_{m n}-\frac{1}{4} H_{m p q} H_{n}{ }^{p q}-\mathcal{T}_{m n}+\nabla_{m} X_{n}+\nabla_{n} X_{m} & =0 \\
\frac{1}{2} \nabla^{p} H_{p m n}+\frac{1}{2} \mathcal{F}^{p} \mathcal{F}_{p m n}+\frac{1}{12} \mathcal{F}_{m n p q r} \mathcal{F}^{p q r}-X^{p} H_{p m n}-\partial_{m} X_{n}+\partial_{n} X_{m} & =0 \\
R-\frac{1}{12} H_{m n p}^{2}+4 \nabla_{m} X^{m}-4 X_{m} X^{m} & =0
\end{aligned}
$$

where $\nabla_{m}$ denotes the space-time covariant derivative, $R_{m n}$ the Ricci tensor, $R$ the Ricci scalar and $H_{m n p}=3 \partial_{[m} B_{n p]}+$ the field strength of the NS-NS $B$-field. The R-R fields enter via the currents $\mathcal{F}_{m_{1} \cdots m_{n}}$ and contribute to the stress tensor in (2.1a) via

$$
\mathcal{T}_{m n}=\frac{1}{2} \mathcal{F}_{m} \mathcal{F}_{n}+\frac{1}{4} \mathcal{F}_{m p q} \mathcal{F}_{n}{ }^{p q}+\frac{1}{4 \times 4 !} \mathcal{F}_{m p q r s} \mathcal{F}_{n}{ }^{p q r s}-\frac{1}{4} G_{m n}\left(\mathcal{F}_{p} \mathcal{F}^{p}+\frac{1}{6} \mathcal{F}_{p q r} \mathcal{F}^{p q r}\right) \text {. }
$$


The equations (2.1a)-(2.1c) are based on the existence of a Killing vector field $K$ and an additional vector field $Z$ with $K^{m} Z_{m}=0$, which enter the field equations in the combination $X \equiv K+Z$. The vector field $Z$ satisfies the Bianchi type equations

$$
\partial_{m} Z_{n}-\partial_{n} Z_{m}+K^{p} H_{p m n}=0
$$

The ordinary type IIB equations are recovered in the limit where $K=0$ such that $Z$ can be integrated to the dilaton field $Z_{m}=\partial_{m} \phi$.

In the R-R sector, the generalized dynamical equations for the field strengths $\mathcal{F}_{m_{1} \cdots m_{n}}$ are given by

$$
\begin{array}{r}
\nabla^{m} \mathcal{F}_{m}-Z^{m} \mathcal{F}_{m}-\frac{1}{6} H^{m n p} \mathcal{F}_{m n p}=0, \quad K^{m} \mathcal{F}_{m}=0, \\
\nabla^{p} \mathcal{F}_{p m n}-Z^{p} \mathcal{F}_{p m n}-\frac{1}{6} H^{p q r} \mathcal{F}_{m n p q r}-\left(K \wedge \mathcal{F}_{1}\right)_{m n}=0, \\
\nabla^{r} \mathcal{F}_{\text {rmnpq }}-Z^{r} \mathcal{F}_{\text {rmnpq }}+\frac{1}{36} \varepsilon_{\text {mnpqrstuvw }} H^{r s t} \mathcal{F}^{u v w}-\left(K \wedge \mathcal{F}_{3}\right)_{m n p q}=0,
\end{array}
$$

while their modified Bianchi identities can be cast into the compact form

$$
d \mathcal{F}_{2 n+1}-Z \wedge \mathcal{F}_{2 n+1}+H_{3} \wedge \mathcal{F}_{2 n-1}=\star\left(K \wedge \star \mathcal{F}_{2 n+3}\right)
$$

The Bianchi identities extend to the dual field strengths $-\mathcal{F}_{7} \equiv \star \mathcal{F}_{3}$ and $\mathcal{F}_{9} \equiv \star \mathcal{F}_{1}$. Furthermore, the selfduality property $\mathcal{F}_{\text {rmnpq }}=\star \mathcal{F}_{\text {rmnpq }}$ of the five form continues to hold in the modified theory, relating its Bianchi identity and field equation. In the following, for simplicity of the formulas, we will often choose coordinates such that the Killing vector field points in a given direction $K^{m}=\delta_{*}^{m}$.

\subsection{Solution of the Bianchi identities}

It has been noted in [28] that equation (2.2) for the new vector $Z_{m}$ may be interpreted as a modified "dilaton Bianchi identity" and locally integrated into

$$
Z_{m}=\partial_{m} \phi+K^{p} B_{p m+}=\partial_{m} \phi-B_{m *+} .
$$

We will in the following stay in this picture and understand the combination $\partial_{m} \phi-B_{m *+}$ as a derivative $D_{m} \phi$ on the dilaton that is covariantized in a suitable sense. As a related observation, one may straightforwardly check that the modified Bianchi identities (2.4) satisfied by the R-R field strengths allow for an explicit integration into $\mathcal{F}=e^{\phi} F$ with

$$
\begin{aligned}
F_{m}= & \partial_{m} \chi+B_{m *+} \chi+B_{m *-} \equiv D_{m} \chi \\
F_{p m n}= & 3 \partial_{[p} B_{m n]-}+\frac{3}{2} B_{[p|*+|} B_{m n]-}-\frac{3}{2} B_{[p|*-|} B_{m n]+}+C_{p m n *}+\chi H_{p m n}, \\
F_{m n p q r}= & 5 \partial_{[m} C_{n p q r]}+5 B_{[m|*+|} C_{n p q r]}-15 B_{[m n|+|} \partial_{p} B_{q r]-} \\
& -15 B_{[m n|+|} B_{p|*+|} B_{q r]-}+15 B_{[m n|-|} \partial_{p} B_{q r]+}+C_{m n p q r *+}, \\
F_{m n p q r s t}= & 7 \partial_{[m} C_{n p q r s t]+}+7 B_{[m *+} C_{n p q r s t]+}+35 C_{[m n p q} H_{r s t]} \\
& -105 B_{[m n|+|} B_{p q|-|} H_{r s t]}+C_{m n p q r s t *} .
\end{aligned}
$$


All the terms, carrying indices "* represent the deformations from the standard IIB expressions. Again, in the following we will assign them a natural interpretation as the connection terms of covariantized derivatives, non-abelian field strengths and the Stückelberg type couplings among $p$-forms. These additional couplings precisely match the structure of general nine-dimensional gauged supergravities $[50,51]$ (recall that due to the existence of a Killing vector field, we are effectively describing a nine-dimensional theory). More precisely, equations (2.6) can be viewed as resulting from a gauging of nine-dimensional maximal supergravity in which a linear combination of the Cartan subgroup of the SL(2) IIB and the trombone symmetry which scales every field according to its Weyl weight has been gauged. The component $B_{m *+}$ of the ten-dimensional NS-NS two-form serves as a gauge field. ${ }^{2}$ An important consequence is the following. According to (2.5), the dilaton $\phi$ is charged under the new local gauge symmetry. Translation to the Einstein frame via

$$
G_{m n}^{\mathrm{st}}=e^{\phi / 2} G_{m n}^{\mathrm{E}},
$$

thus implies that the metric in the Einstein frame is also charged. Translation of the Einstein field equations (2.1a) into the Einstein frame thus induces field equations which feature a covariantized Ricci tensor in the sense that all derivatives in its definition are replaced by properly covariantized ones. In particular, the Riemann tensor is calculated as curvature of the connection

$$
\hat{\Gamma}_{m n}^{p} \equiv \frac{1}{2} G^{p q}\left(D_{m} G_{n q}+D_{n} G_{m q}-D_{q} G_{m n}\right), \quad D_{p} G_{m n} \equiv \partial_{p} G_{m n}+\frac{1}{2} B_{p *+} G_{m n} .
$$

This is the generic structure of supergravities in which the trombone symmetry is gauged [52]. Upon transition to the Einstein frame, we may also regroup the field equations for NS-NS and R-R two-form (2.1b) and (2.3b) into the manifestly SL(2) covariant form

$$
D_{p}\left(F^{p m n \alpha} m_{\alpha \beta}\right)-\frac{1}{6} F^{m n p q r} F_{p q r}^{\alpha} \varepsilon_{\alpha \beta}=J_{\beta}^{m n},
$$

with the SL(2) doublet $F_{m n p}=\left\{H_{m n p}, F_{m n p}-\chi H_{m n p}\right\}$, and the dilaton/axion matrix $m_{\alpha \beta}$ parametrized as

$$
m_{\alpha \beta}=\left(\begin{array}{cc}
e^{\phi} & -e^{\phi} \chi \\
-e^{\phi} \chi & e^{\phi} \chi^{2}+e^{-\phi}
\end{array}\right) .
$$

The current on the r.h.s. of (2.9) is given by the $\mathrm{SL}(2)$ doublet

$$
J_{ \pm}^{m n}=\left\{2 e^{2 \phi} K^{[m} F^{n]},-4 e^{2 \phi} \nabla^{[m} K^{n]}-2 \chi e^{2 \phi} K^{[m} F^{n]}\right\},
$$

in terms of the Killing vector field $K^{m}$ and the current $F_{m}=D_{m} \chi$. We will in the following recover the non-abelian field-strengths (2.6) from a particular Scherk-Schwarz ansatz in exceptional field theory.

\footnotetext{
${ }^{2}$ To be precise, also a nilpotent generator of $\mathrm{SL}(2)_{\text {IIB }}$ is gauged with the component $B_{m *-}$ serving as the associated gauge field.
} 


\section{Exceptional field theory}

\section{$3.1 \quad \mathbf{E}_{6(6)}$ exceptional field theory}

In this section, we give a brief review of the exceptional field theory associated with the group $\mathrm{E}_{6(6)}$ into which we will in the following embed the generalized IIB supergravity equations. We refer to $[42,44,53]$ for details of the theory. The bosonic field content of $\mathrm{E}_{6(6)}$ exceptional field theory reflects the field content of maximal $D=5$ supergravity, and is given by

$$
\left\{g_{\mu \nu}, \mathcal{M}_{M N}, \mathcal{A}_{\mu}^{M}, \mathcal{B}_{\mu \nu M}\right\}
$$

with indices $\mu, \nu=0, \ldots, 4$, and $M=1, \ldots, 27$. The symmetric matrices $g_{\mu \nu}$ and $\mathcal{M}_{M N}$ define the external and internal metrics, respectively, the latter parametrizing the coset space $\mathrm{E}_{6(6)} / \mathrm{USp}(8)$. In addition to their dependence on the five external coordinates $\left\{x^{\mu}\right\}$, the fields (3.1) formally depend on 27 internal coordinates $\left\{Y^{M}\right\}$ transforming in the fundamental representation of $\mathrm{E}_{6(6)}$. The latter dependence is strongly restricted by the $\mathrm{E}_{6(6)}$ covariant section condition $[54,55]$ (on any two fields or gauge parameters $A, B$ )

$$
d^{K M N} \partial_{M} \partial_{N} A=0, \quad d^{K M N} \partial_{M} A \partial_{N} B=0,
$$

with $d^{K M N}$ denoting the totally symmetric cubic invariant of $\mathrm{E}_{6(6)}$. The constraints (3.2) admit two inequivalent solutions, in which the fields depend on a subset of six or five of the internal coordinates. Upon implementing one of these solutions, the field equations of exceptional field theory reproduce full $D=11$ supergravity and ten-dimensional IIB supergravity, respectively.

The field equations of exceptional field theory are most compactly obtained from variation of a Lagrangian

$$
\begin{aligned}
\sqrt{|g|}^{-1} \mathcal{L}_{\mathrm{ExFT}}= & \widehat{R}+\frac{1}{24} g^{\mu \nu} D_{\mu} \mathcal{M}_{M N} D_{\nu} \mathcal{M}^{M N}-\frac{1}{4} \mathcal{F}_{\mu \nu}{ }^{M} \mathcal{F}^{\mu \nu N} \mathcal{M}_{M N} \\
& +\sqrt{|g|}{ }^{-1} \mathcal{L}_{\text {top }}-V_{\text {pot }} .
\end{aligned}
$$

The first term formally takes the same form as the five-dimensional Einstein-Hilbert term, where in the definition of the Ricci scalar $\widehat{R}$ all external derivatives are covariantized w.r.t. the action of internal diffeomorphisms (under which the external metric transforms as a weighted scalar)

$$
\partial_{\mu} g_{\nu \rho} \longrightarrow D_{\mu} g_{\nu \rho} \equiv \partial_{\mu} g_{\nu \rho}-\mathcal{A}_{\mu}{ }^{M} \partial_{M} g_{\nu \rho}-\frac{2}{3} \partial_{M} \mathcal{A}_{\mu}{ }^{M} g_{\nu \rho} .
$$

The second term in (3.3) is a gauged coset space sigma-model $\mathrm{E}_{6(6)} / \mathrm{USp}(8)$ with derivatives $D_{\mu} \equiv \partial_{\mu}-\mathcal{L}_{\mathcal{A}_{\mu}}$ covariantized under the action of generalized internal diffeomorphisms [55]

$$
\begin{aligned}
\mathcal{L}_{\Lambda} \mathcal{M}_{M N}= & \Lambda^{K} \partial_{K} \mathcal{M}_{M N}+2 \partial_{(M} \Lambda^{K} \mathcal{M}_{N) K}-10 \partial_{K} \Lambda^{L} d^{K P R} d_{R L(M} \mathcal{M}_{N) P} \\
& +\frac{2}{3} \partial_{P} \Lambda^{P} \mathcal{M}_{M N} .
\end{aligned}
$$


The third term in (3.3) describes a Yang-Mills type kinetic term for the 27 gauge vectors $\mathcal{A}_{\mu}{ }^{M}$ whose non-abelian structure again is induced by the structure of internal diffeomorphisms

$$
\begin{aligned}
\mathcal{F}_{\mu \nu}{ }^{M}= & 2 \partial_{[\mu} \mathcal{A}_{\nu]}{ }^{M}-2 \mathcal{A}_{[\mu}{ }^{K} \partial_{K} \mathcal{A}_{\nu]}{ }^{M}+10 d^{M K R} d_{N L R} \mathcal{A}_{[\mu}{ }^{N} \partial_{K} \mathcal{A}_{\nu]}{ }^{L} \\
& +10 d^{M N K} \partial_{K} \mathcal{B}_{\mu \nu N} .
\end{aligned}
$$

The Stückelberg-type coupling to the 27 two-forms $\mathcal{B}_{\mu \nu N}$ induces a modification of the Bianchi identities as

$$
3 D_{[\mu} \mathcal{F}_{\nu \rho]}^{M}=10 d^{M N K} \partial_{K} \mathcal{H}_{\mu \nu \rho N},
$$

with the 3 -form field strength $\mathcal{H}_{\mu \nu \rho N}$ defined by this equation (up to terms that vanish under projection with $d^{M N K} \partial_{K}$ ). The term $\mathcal{L}_{\text {top }}$ in (3.3) refers to a Chern-Simons-type topological term which is such that the field equations obtained by varying the full Lagrangian w.r.t. the two-forms $\mathcal{B}_{\mu \nu M}$ give rise to the first order duality equations

$$
d^{M N K} \partial_{K}\left(\sqrt{|g|} \mathcal{M}_{N L} \mathcal{F}^{\mu \nu L}+\frac{1}{6} \sqrt{10} \varepsilon^{\mu \nu \rho \sigma \tau} \mathcal{H}_{\rho \sigma \tau N}\right)=0
$$

relating the field strengths of vector fields and two-forms. Finally, the last term in (3.3) is the 'scalar potential' that involves only internal derivatives $\partial_{M}$ whose explicit form can be found in [42]. Its form is uniquely determined by invariance under internal generalized diffeomorphisms.

\subsection{Section constraints and IIA/IIB/generalized supergravity}

The section condition (3.2) is solved by restricting the internal coordinate dependence of all fields to properly chosen subsets of coordinates. Breaking $\mathrm{E}_{6(6)}$ down to its subgroup $\mathrm{SL}(5) \times \mathrm{SL}(2) \times \mathrm{GL}(1)$ IIB according to

$$
\begin{aligned}
\mathbf{2 7} & \longrightarrow(5,1)_{+4}+\left(5^{\prime}, 2\right)_{+1}+(10,1)_{-2}+(1,2)_{-5}, \\
\left\{Y^{M}\right\} & \longrightarrow\left\{y^{a}, \tilde{y}_{a \alpha}, \tilde{y}^{a b}, \tilde{y}_{\alpha}\right\}, \quad a=1, \ldots, 5, \quad \alpha= \pm,
\end{aligned}
$$

and restricting all fields to depend only on the 5 coordinates $\left\{y^{a}\right\}$ of highest grading under GL(1) IIB solves the conditions (3.2). The ExFT field equations derived from (3.3) then reproduce the IIB theory after decomposing the ExFT fields (3.1) according to (3.9) and properly translating the various blocks into the various components of the IIB fields [44]. In particular, the scalar matrix $\mathcal{M}_{M N}$ decomposes into

$$
\mathcal{M}_{K M}=\left(\begin{array}{cccc}
\mathcal{M}_{a c} & \mathcal{M}_{a}{ }^{c \beta} & \mathcal{M}_{a, c d} & \mathcal{M}_{a}{ }^{\beta} \\
\mathcal{M}^{a \alpha}{ }_{c} & \mathcal{M}^{a \alpha, c \beta} & \mathcal{M}^{a \alpha}{ }_{c d} & \mathcal{M}^{a \alpha, \beta} \\
\mathcal{M}_{a b, c} & \mathcal{M}_{a b}{ }^{c \beta} & \mathcal{M}_{a b, c d} & \mathcal{M}_{a b}{ }^{\beta} \\
\mathcal{M}^{\alpha}{ }_{c} & \mathcal{M}^{\alpha, c \beta} & \mathcal{M}^{\alpha}{ }_{c d} & \mathcal{M}^{\alpha \beta}
\end{array}\right),
$$

where the explicit form of the blocks is obtained by evaluating the matrix $\mathcal{M}_{M N}=$ $\mathcal{V}_{M}{ }^{A} \mathcal{V}_{N}{ }^{A}$ from a vielbein $\mathcal{V}_{M}{ }^{A}$ given by the product of matrix exponentials

$$
\mathcal{V}_{\mathrm{IIB}} \equiv \exp \left[\varepsilon^{a b c d e} c_{a b c d} t_{(+4) e}\right] \exp \left[b_{a b}{ }^{\alpha} t_{(+2) \alpha}^{a b}\right] \mathcal{V}_{5} \mathcal{V}_{2} \exp \left[\Phi t_{\mathrm{IIB}}\right]
$$


with the relevant $\mathfrak{e}_{6(6)}$ generators $t_{\mathrm{IIB}}, t_{(+2) \alpha}^{a b}, t_{(+4) a}$ and their coefficients originating from the IIB metric, two-form and four-form, respectively. The matrices $\mathcal{V}_{5}, \mathcal{V}_{2}$ represent the $\mathrm{SL}(5) \times \mathrm{SL}(2)$ factor of the vielbein, related to the internal metric and the IIB dilaton/axion matrix, respectively. Similarly, vectors and two-forms are decomposed as (3.9)

$$
\begin{aligned}
\left\{\mathcal{A}_{\mu}{ }^{M}\right\} & \longrightarrow\left\{\mathcal{A}_{\mu}{ }^{a}, \mathcal{A}_{\mu a \alpha}, \mathcal{A}_{\mu}{ }^{a b}, \mathcal{A}_{\mu \alpha}\right\} \\
\left\{\mathcal{B}_{\mu \nu M}\right\} & \longrightarrow\left\{\mathcal{B}_{\mu \nu a}, \mathcal{B}_{\mu \nu}{ }^{a \alpha}, \mathcal{B}_{\mu \nu a b}, \mathcal{B}_{\mu \nu \alpha}\right\}
\end{aligned}
$$

In contrast, the IIA theory is recovered, if the physical coordinates are identified with the $\left\{\tilde{y}_{a+}\right\}$ in the decomposition (3.9) (which explicitly breaks the SL(2) factor), the ExFT fields are decomposed accordingly and translated into the IIA fields. E.g. in this case, the proper parametrization of the matrix $\mathcal{M}_{M N}=\mathcal{V}_{M}{ }^{A} \mathcal{V}_{N}{ }^{A}$ is obtained via a vielbein $\mathcal{V}_{M}{ }^{A}$

$$
\mathcal{V}_{\mathrm{IIA}} \equiv \exp \left[\varphi t_{(+5)}\right] \exp \left[c_{a b c} t_{(+3)}^{a b c}\right] \exp \left[b_{a b} t_{(+2)}^{a b}\right] \exp \left[c_{a} t_{(+1)}^{a}\right] \mathcal{V}_{5} \exp \left[\phi t_{0}+\Phi t_{\text {IIA }}\right]
$$

with the coefficients originating from the IIA metric, dilaton, and $p$-forms, respectively.

In this paper we choose yet a different solution of the section constraint. First, we impose the existence of a Killing vector field in the IIB theory and accordingly split the coordinates $\left\{y^{a}\right\}=\left\{y^{i}, y^{*}\right\},(i=1, \ldots, 4)$, such that $\partial_{*} \Phi=0$ for all fields of the theory. Next, we relax the IIB solution, by allowing fields to depend on the 5 coordinates

$$
\left\{y^{i}, \tilde{y}_{*+}\right\}, \quad i=1, \ldots, 4
$$

such that the section condition (3.2) is still satisfied. In the following, we will evaluate ExFT in the IIB parametrization (3.10) however imposing a particular additional $\tilde{y}_{*+}-$ dependence according to a simple Scherk-Schwarz ansatz which will trigger the generalized IIB equations. It is important to note that the choice of coordinates (3.14) is equivalent (after rotation of the 27 coordinates) to selecting the IIA coordinates $\tilde{y}_{a+}$ in (3.9). Applying the same rotation to the IIA parametrization of ExFT fields such as (3.13) we would simply recover the IIA theory. This is a manifestation of the fact that the generalized IIB supergravity equations can be obtained via T-duality from a sector of IIA supergravity. Since the framework of exceptional field theory is manifestly duality covariant, we can simply absorb the effect of this duality into a rotation of the extended coordinates. We will thus evaluate exceptional field theory in its IIB parametrization (3.10) however in coordinates (3.14) and with a proper Scherk-Schwarz ansatz in $\tilde{y}_{*+}$ in order to obtain directly the generalized IIB equations.

\subsection{Scherk-Schwarz ansatz}

Following the previous discussion, and having chosen physical coordinates according to (3.14) we now impose on the ExFT fields (3.1) a specific $\tilde{y}_{*+}$-dependence, such that in particular the total $\tilde{y}_{*+}$-dependence consistently factors out from all the equations of 
motion. This is achieved by a Scherk-Schwarz ansatz [56]

$$
\begin{aligned}
\mathcal{M}_{M N} & =U_{M}^{K}(\tilde{y}) U_{N}^{L}(\tilde{y}) M_{K L}\left(x^{\mu}, y^{i}\right), \\
g_{\mu \nu} & =\rho^{-2}(\tilde{y}) g_{\mu \nu}\left(x^{\mu}, y^{i}\right) \\
\mathcal{A}_{\mu}{ }^{M} & =\rho^{-1}(\tilde{y}) A_{\mu}{ }^{N}\left(x^{\mu}, y^{i}\right)\left(U^{-1}\right)_{N}^{M}(\tilde{y}), \\
\mathcal{B}_{\mu \nu M} & =\rho^{-2}(\tilde{y}) U_{M}^{N}(\tilde{y}) B_{\mu \nu N}\left(x^{\mu}, y^{i}\right),
\end{aligned}
$$

where the $\tilde{y}_{*+-}$-dependence of all fields is carried by an $\mathrm{E}_{6(6)}$-valued twist matrix $U_{N}{ }^{L}$ and a scalar factor $\rho$. For simplicity of the notation, here and in the following we also use the notation $\tilde{y} \equiv \tilde{y}_{*+} \cdot{ }^{3}$

The relevant Scherk-Schwarz ansatz for generalized IIB supergravity is based on a twist matrix $U_{M}^{N}$ living in an

$$
\mathrm{GL}(1) \subset \mathrm{SL}(2)_{\operatorname{diag}} \subset \mathrm{SL}(2) \times \mathrm{SL}(2) \subset \mathrm{SL}(2) \times \mathrm{SL}(6) \subset \mathrm{E}_{6(6)},
$$

subgroup of the full duality group $\mathrm{E}_{6(6)}$. More precisely, upon decomposing

$$
\begin{aligned}
\mathrm{E}_{6(6)} & \rightarrow \mathrm{SL}(2) \times \mathrm{SL}(6), \\
\mathbf{2 7} & \longrightarrow(1,15)+\left(2,6^{\prime}\right), \quad\left\{Y^{M}\right\} \rightarrow\left\{Y^{\hat{a} \hat{b}}, \tilde{Y}_{\hat{a} \alpha}\right\},
\end{aligned}
$$

an $(\mathrm{SL}(2) \times \mathrm{SL}(6))$-valued matrix $U$ takes the form

$$
U_{M}^{N}=\left(\begin{array}{cc}
U_{\hat{a}}^{[\hat{c}} U_{\hat{b}}^{\hat{d}]} & 0 \\
0 & \left(U^{-1}\right)_{\hat{a}}^{\hat{c}} U_{\alpha}^{\beta}
\end{array}\right),
$$

and we choose the matrix factors as

$$
\begin{aligned}
U_{\alpha}{ }^{\beta} & =\left(\begin{array}{cc}
U_{+}^{+} & 0 \\
0 & U_{-}^{-}
\end{array}\right)=\left(\begin{array}{cc}
\rho(\tilde{y}) & 0 \\
0 & \rho^{-1}(\tilde{y})
\end{array}\right), \\
U_{\hat{a}}^{\hat{b}} & =\left(\begin{array}{ccc}
U_{i}^{j} & 0 & 0 \\
0 & U_{*}^{*} & 0 \\
0 & 0 & U_{0}{ }^{0}
\end{array}\right)=\left(\begin{array}{ccc}
\delta_{i}^{j} & 0 & 0 \\
0 & \rho(\tilde{y}) & 0 \\
0 & 0 & \rho^{-1}(\tilde{y})
\end{array}\right),
\end{aligned}
$$

with scale factor given by a linear function $\rho(\tilde{y})=\tilde{y}+c$. In order to check the effect of the Scherk-Schwarz ansatz (3.15) with (3.19) on the field equations of exceptional field theory, we consider the current

$$
\left(\mathcal{X}_{M}\right)_{N}{ }^{K} \equiv \rho^{-1}\left(U^{-1}\right)_{M}{ }^{P}\left(U^{-1}\right)_{N}{ }^{Q} \partial_{P} U_{Q}{ }^{K},
$$

\footnotetext{
${ }^{3}$ Note that the ansatz (3.15) is slightly more general than the ones studied in [56] in that the fields multiplying the twist matrices on the r.h.s. do not only depend on the external coordinates $x^{\mu}$ but also on part of the internal coordinates $y^{i}$. In this sense, the ansatz (3.15) resembles the embedding of deformations of ExFT studied in [57] (and in [58] in the context of double field theory), although here all fields and twist matrices respect the section constraint, so we remain within the original framework.
} 
which encodes the combinations of the twist matrix and its derivatives that explicitly enter the field equations. With the explicit form of (3.19), this current lives in the algebra $\mathfrak{s l}(2) \oplus \mathfrak{s l}(6)$ with its only non-vanishing components given by

$$
\left(\mathcal{X}^{*+}\right)_{\alpha}{ }^{\beta}=\left(\begin{array}{cc}
1 & 0 \\
0 & -1
\end{array}\right), \quad\left(\mathcal{X}^{*+}\right)_{\hat{a}}^{\hat{b}}=\left(\begin{array}{ccc}
0_{4 \times 4} & 0 & 0 \\
0 & 1 & 0 \\
0 & 0 & -1
\end{array}\right)
$$

all constant, ensuring that the $\tilde{y}$-dependence factors out from all equations of motion. ${ }^{4}$ We have thus presented a consistent Scherk-Schwarz ansatz on the ExFT fields which moreover satisfies the section condition. Upon explicitly evaluating the field equations, the non-trivial $\tilde{y}$ dependence of the twist matrix induces a deformation of the original IB equations of motion. We shall work this out in the next section.

\subsection{Induced deformation}

In this section we will illustrate with several examples how the Scherk-Schwarz ansatz (3.15) induces a deformation of the resulting field equations which precisely coincides with the deformation of the IIB field equations and Bianchi identities discussed in section 2 above. Covariant derivatives in ExFT carry vector fields $\mathcal{A}_{\mu}{ }^{M}$ and internal derivatives $\partial_{M}$. Under $(\mathrm{SL}(2) \times \mathrm{SL}(6))$, the coordinates (3.14) are embedded in the $Y^{M}$ as $\left\{Y^{i 0}, \tilde{Y}_{*+}\right\}$, cf. (3.17). With the ansatz (3.15), the relevant couplings then are obtained from

$$
\mathcal{A}_{\mu}{ }^{i 0} \partial_{i 0}=\rho^{-1} \rho A_{\mu}^{i 0} \partial_{i 0}, \quad \mathcal{A}_{\mu *+} \partial^{*+}=\rho^{-1} \rho^{2} A_{\mu *+} \partial^{*+} .
$$

Both operators give rise to additional $\tilde{y}$-independent couplings. Let us e.g. consider the covariant derivative on the external metric (3.4). With the Scherk-Schwarz ansatz (3.15), we obtain via $(3.22)$

$$
D_{\mu} g_{\nu \rho}=\rho^{-2}(\tilde{y})\left(\partial_{\mu} \mathrm{g}_{\nu \rho}-2 A_{\mu}{ }^{i 0} \partial_{i 0} \mathrm{~g}_{\nu \rho}-\frac{4}{3} \partial_{0 i} A_{\mu}{ }^{0 i} \mathrm{~g}_{\nu \rho}+\frac{4}{3} A_{\mu *+} \mathrm{g}_{\nu \rho}\right) .
$$

The first three terms on the r.h.s. correspond to the standard ExFT result and upon translation into the IIB fields contribute to the standard IIB field equations [44]. We will thus employ the notation

$$
D_{\mu} g_{\nu \rho}=\rho^{-2}(\tilde{y})\left(\stackrel{\circ}{D}_{\mu} \mathrm{g}_{\nu \rho}+\frac{4}{3} A_{\mu *+} \mathrm{g}_{\nu \rho}\right)
$$

The last term captures the effect of the Scherk-Schwarz twist matrix and shows that the IIB space-time metric acquires non-trivial covariant derivatives which is precisely in accordance with our discussion above regarding the charged IIB metric (2.8) after transition to the

\footnotetext{
${ }^{4}$ Strictly speaking, for consistency of the Scherk-Schwarz ansatz a weaker condition is sufficient: only the projection of (3.20) onto the $\mathbf{2 7} \oplus \mathbf{3 5 1}$ representation of $\mathrm{E}_{6(6)}$ appears in the field equations and is required to be constant. With (3.21) this is automatically guaranteed.
} 
Einstein frame. ${ }^{5}$ The Riemann tensor whose contraction appears in the Einstein field equations will thus correspond to the curvature of the modified connection (2.8) as in the generalized IIB equations.

In a similar way, we can work out the ExFT field strengths (3.6) under the Scherk-Schwarz ansatz (3.15). As a general feature of the Scherk-Schwarz ansatz with consistent twist matrices, the $\tilde{y}$-dependence of these field strengths consistently factors out according to

$$
\mathcal{F}_{\mu \nu}^{M}(x, Y)=\rho^{-1}(\tilde{y})\left(U^{-1}\right)_{N}^{M}(\tilde{y}) \mathcal{F}_{\mu \nu}^{M}(x, y)
$$

where

$$
\mathcal{F}_{\mu \nu}{ }^{M}(x, y) \equiv \stackrel{\circ}{\mathcal{F}}_{\mu \nu}{ }^{N M}+X_{K L}{ }^{M}\left(A_{[\mu}{ }^{K} A_{\nu]}^{L}-2 d^{K L N} B_{\mu \nu N}\right)
$$

describes a deformation of the standard ExFT field strength $\stackrel{\circ}{\mathcal{F}}_{\mu \nu}{ }^{M}$ by non-abelian terms carrying the generic structure of five-dimensional gauged supergravity [59] encoded in an embedding tensor $X_{M N}{ }^{K}$ living in the $\mathbf{3 5 1}+\mathbf{2 7}$ representation of $\mathrm{E}_{6(6)}$. Within the Scherk-Schwarz ansatz, the embedding tensor is obtained from projecting (3.20) onto the relevant $\mathrm{E}_{6(6)}$ representations. Again, the form of (3.27) resembles the deformations of ExFT studied in [57], although here it simply results from a Scherk-Schwarz ansatz within the original ExFT. Structurewise, the new couplings (3.27) resemble those introduced in (2.6) in order to account for the deformed Bianchi identities in generalized IIB supergravity. In the rest of this paper, we will make the agreement precise using the explicit dictionary between ExFT and IIB fields [44].

Working out (3.6), it follows that the twist matrix (3.18)-(3.19) induces an embedding tensor

$$
X_{M N}{ }^{K}=\left(\tilde{X}_{M}\right)_{N}{ }^{K}+\frac{2}{3} \delta_{M}{ }^{*+} \delta_{N}{ }^{K},
$$

in (3.27). Upon contraction with a gauge parameter $\Lambda^{M}$ it identifies the gauged generators within $\mathfrak{e}_{6(6)} \oplus \mathbb{R}_{\text {tromb. }}$. The second term in (3.28) refers to the gauging of the trombone symmetry under which the ExFT fields $\left\{g_{\mu \nu}, \mathcal{M}_{M N}, \mathcal{A}_{\mu}{ }^{M}, \mathcal{B}_{\mu \nu M}\right\}$ scale with weight $\{2,0,1,2\}$, respectively, whose effect we have already observed in (3.24). The first term in (3.28) identifies the gauged generators within $\mathfrak{e}_{6(6)}$, combining the diagonal generators

$$
\left(\Lambda^{M} \tilde{X}_{M}\right)_{\mathfrak{s l}(2)}=\left(\begin{array}{cc}
\frac{1}{2} \Lambda_{*+} & 0 \\
-\Lambda_{*-} & -\frac{1}{2} \Lambda_{*+}
\end{array}\right), \quad\left(\Lambda^{M} \tilde{X}_{M}\right)_{\mathfrak{s l}(6)}=\left(\begin{array}{ccc}
\frac{1}{6} \Lambda_{*+} \mathbb{I}_{4} & 0 & 0 \\
0 & \frac{1}{6} \Lambda_{*+} & 0 \\
0 & \Lambda_{0+} & -\frac{5}{6} \Lambda_{*+}
\end{array}\right)
$$

\footnotetext{
${ }^{5}$ To be precise, after identification $A_{\mu *+}=B_{\mu *+}$, the factor $4 / 3$ in (3.24) comes via the standard $5+5$ Kaluza-Klein decomposition

$$
G_{m n}=\left(\begin{array}{cc}
\left(\operatorname{det} g_{a b}\right)^{-1 / 3} g_{\mu \nu}+\ldots & A_{\mu}{ }^{b} g_{a b} \\
g_{a b} A_{\mu}{ }^{b} & g_{a b}
\end{array}\right)
$$

of the IIB metric.
} 
within $\mathfrak{s l}(2) \oplus \mathfrak{s l}(6)$ with the off-diagonal generators

$$
\begin{aligned}
& \left(\Lambda^{M} \tilde{X}_{M}\right)^{*+, i j}=\left(\Lambda^{M} \tilde{X}_{M}\right)^{i+, j *}=-\Lambda^{i j}, \\
& \left(\Lambda^{M} \tilde{X}_{M}\right)_{i j, 0-}=\left(\Lambda^{M} \tilde{X}_{M}\right)_{0 i, j-}=-\frac{1}{2} \varepsilon_{* i j k l} \Lambda^{k l},
\end{aligned}
$$

in $\mathfrak{e}_{6(6)} \backslash(\mathfrak{s l}(6) \oplus \mathfrak{s l}(2))$. The Stückelberg-type couplings in (3.27) to the two-forms $B_{\mu \nu} M$ are read off from (3.29), (3.30) together with the explicit form of $d^{M N P}$ in the decomposition (3.9), see [44]. The explicit result for the various components of the field strengths (3.27) is the following

$$
\begin{aligned}
\mathcal{F}_{\mu \nu a+} & =\stackrel{\circ}{\mathcal{F}}_{\mu \nu i+}, \\
\mathcal{F}_{\mu \nu a-} & =\stackrel{\circ}{\mathcal{F}}_{\mu \nu a-}+A_{[\mu *+} A_{\nu] a-}-A_{[\mu *-} A_{\nu] a+}+\sqrt{2} \tilde{B}_{\mu \nu a *}, \\
\mathcal{F}_{\mu \nu a b c} & =\stackrel{\mathcal{F}}{\mu \nu}_{\mu \nu a b c}+2 A_{[\mu *+} A_{\nu] a b c}+\frac{3}{2 \sqrt{2}} \varepsilon_{a b c d *} \tilde{B}_{\mu \nu}{ }^{d-}, \\
\mathcal{F}_{\mu \nu+} & =\stackrel{\circ}{\mathcal{F}}_{\mu \nu+}+2 A_{[\mu *+} A_{\nu]+},
\end{aligned}
$$

with the redefined two-forms

$$
\begin{aligned}
\tilde{B}_{\mu \nu a b} & \equiv \sqrt{10} B_{\mu \nu a b}+A_{[\mu}^{c} A_{\nu] a b c}, \\
\tilde{B}_{\mu \nu}^{k \alpha} & \equiv \sqrt{10} B_{\mu \nu}^{a \alpha}+\varepsilon^{\alpha \beta} A_{[\mu}^{a} A_{\nu] \beta}+\frac{\sqrt{2}}{6} \varepsilon^{\alpha \beta} \varepsilon^{a b c d e} A_{[\mu|b \beta|} A_{\nu] c d e} .
\end{aligned}
$$

Comparing the deformed field strengths (3.31) to the field strengths (2.6) solving the Bianchi identities of generalized IIB supergravity, we find precise agreement upon identifying the ExFT components with the IIB field strengths (the precise dictionary between fields has been given in [44] and in particular takes care of the $\sqrt{2}$ factors that arise in the ExFT expressions (3.31)).

Of course, the field strengths (3.31) only represent part of the full IIB field strengths, in which two of the ten-dimensional indices are chosen to be external. The remaining IIB components will appear among other ExFT fields. E.g. let us consider the three-form field strength $\mathcal{H}_{\mu \nu \rho M}$ defined by (3.7). Evaluating this definition with the above Scherk-Schwarz ansatz in particular yields the components

$$
\begin{aligned}
\mathcal{H}_{\mu \nu \rho-} & =\stackrel{\mathcal{H}}{\mu \nu \rho-}+\sqrt{2} \mathcal{O}_{\mu \nu \rho} \\
\mathcal{H}_{\mu \nu \rho * a} & =\stackrel{\mathcal{H}}{\mu \nu \rho * i}+3 A_{[\mu|*+|} \tilde{B}_{\nu \rho] * a}+\frac{3 \sqrt{2}}{2} A_{[\mu|*+|} A_{\nu|*-|} A_{\rho] a+}-\partial_{a} \mathcal{O}_{\mu \nu \rho} .
\end{aligned}
$$

The second and third term of $\mathcal{H}_{\mu \nu \rho * i}$ reproduce the corresponding deformation terms in (2.6). The term $\mathcal{O}_{\mu \nu \rho}$ in (3.33) denotes the undetermined contribution in the field strength which vanishes under the projection $d^{K M N} \partial_{N}$ in (3.7). In the undeformed IIB theory, this term is already present in $\mathcal{H}_{\mu \nu \rho * i}$. It arises as an integration constant in the ExFT field equations and is identified with a component of the IIB four-form according to

$$
\sqrt{2} \mathcal{O}_{\mu \nu \rho}=C_{\mu \nu \rho *}+\frac{3}{2} B_{[\mu|*+|} B_{\nu \rho]-}-\frac{3}{2} B_{[\mu|*-|} B_{\nu \rho]+},
$$


in order to reconstruct the selfdual IIB five-form field strength from ExFT. In the deformed case we are considering here, the same $\mathcal{O}_{\mu \nu \rho}$ arises as part of $\mathcal{H}_{\mu \nu \rho-}$ in (3.33) where it precisely acounts for the deformation of the IIB three-form field strength $F_{\mu \nu \rho}$, see (2.6). Again we thus find complete agreement.

In a similar way, the deformed scalar currents $\mathcal{M}^{M K} D_{\mu} \mathcal{M}_{K N}$ with the block decomposition (3.10) and parametrization (3.11) can be matched to the corresponding components of (2.6) in which one of the ten-dimensional indices is chosen to be external. Thus all the building blocks of the ExFT Lagrangian (3.3) exhibit precisely the deformations of their IIB counterparts (2.6). Since equations (2.6) were derived as solution of the deformed IIB Bianchi identities, it follows that after imposing the Scherk-Schwarz ansatz (3.15), the ExFT fields satisfy the deformed IIB Bianchi identities. Moreover, most of the generalized IIB field equations are obtained by covariantization of the standard IIB equations, i.e. by replacing the IIB field strengths by their deformed expressions (2.6). This is true for the Einstein field equations (upon taking into account the charged metric in the Einstein frame, cf. (2.8)) and the self-duality equation $\mathcal{F}_{\text {rmnpq }}=\star \mathcal{F}_{\text {rmnpq }}$ for the five-form field strength. Upon using the explicit dictionary between ExFT fields and IIB fields [44] these equations thus follow from the ExFT dynamics after imposing the Scherk-Schwarz ansatz. The two-form field equations (2.9) in generalized IIB supergravity on the other hand are not only covariantized via (2.6) but also acquire a source term $J^{m n}{ }_{\beta}$. In ExFT, the analogous term descends from variation of the Lagrangian (3.3) w.r.t. the gauge fields which upon implementing the Scherk-Schwarz ansatz gives rise to additional source terms from the Einstein-Hilbert term and the scalar kinetic term.

\section{Conclusions}

In this paper, we have shown how the equations of generalized IIB supergravity found in [28] can naturally be obtained from exceptional field theory upon imposing a simple ScherkSchwarz type ansatz on all the fields that captures their non-isometric behavior in the IIA theory. The Scherk-Schwarz ansatz satisfies the consistency equations [56] and moreover the section constraints (3.2) and induces a deformation of the standard IIB supergravity equations. We have verified explicitly for most of their components that the deformed ExFT fields coincide with the deformed IIB field strengths (2.6) which have been determined by solving the deformed IIB Bianchi identities. The Scherk-Schwarz ansatz straightforwardly extends to the fermionic extension of ExFT [53]. It should thus also be possible to reproduce from exceptional field theory the fermionic completion of the generalized IIB equations that has been worked out in [26]. We should stress that although exceptional field theory admits a Lagrangian formulation (3.3) this does not allow to conclude the existence of an action underlying the generalized IIB equations, since the Scherk-Schwarz ansatz (3.15) is imposed on the level of the field equations and not on the action. The appearance of a trombone gauging (3.28) in the ExFT formulation is in fact a sign that the resulting field equations cannot be obtained from an action [52].

We have in this article embedded the generalized IIB equations into $\mathrm{E}_{6(6)}$ ExFT. In principle, the same construction can be repeated for any of the exceptional field theories 
upon an appropriate decomposition of the IIB coordinates into external and internal coordinates. If one is merely interested in reproducing the generalized IIB equations from a covariant framework, the most efficient framework is the SL(2) ExFT from [60]. In this case, 9-dimensional covariance is kept manifest throughout the construction and other than splitting off the 10th coordinate $y^{*}$, we avoid the split into internal $\left\{y^{i}\right\}$ and external $\left\{x^{\mu}\right\}$ coordinates. Here, we have given the embedding into $\mathrm{E}_{6(6)}$ ExFT since it is this formulation which most naturally carries the $A d S_{5} \times S^{5}$ background in the undeformed case. In particular, in $\mathrm{E}_{6(6)}$ ExFT it follows from a Scherk-Schwarz reduction ansatz that the fluctuations around the $A d S_{5} \times S^{5}$ background give rise to a consistent truncation of IIB supergravity to a five-dimensional maximal supergravity theory $[56,61]$. It will be very interesting to check if similarly the $\mathrm{ABF}$ background allows for a compact formulation within $\mathrm{E}_{6(6)}$ ExFT, combining the Scherk-Schwarz ansatz (3.19) with a deformation of the Scherk-Schwarz twist underlying the $S^{5}$ geometry and an $x^{\mu}$-dependent solution of a five-dimensional supergravity. In particular, this would allow to address the question of consistent truncations around the ABF background and be of particular interest in view of possible holographic interpretations of the deformation. We hope to come back to these questions in the near future.

\section{Acknowledgments}

We would like to thank Riccardo Borsato, Olaf Hohm and Mario Trigiante for helpful discussions. H.S. thanks the Galileo Galilei Institute for Theoretical Physics for the hospitality and the INFN for partial support during the completion of this work. This work is partially supported by the French Agence Nationale de la Recherche (ANR) under grant ANR-15-CE31-0006 DefIS.

Open Access. This article is distributed under the terms of the Creative Commons Attribution License (CC-BY 4.0), which permits any use, distribution and reproduction in any medium, provided the original author(s) and source are credited.

\section{References}

[1] J.M. Maldacena, The large- $N$ limit of superconformal field theories and supergravity, Int. J. Theor. Phys. 38 (1999) 1113 [hep-th/9711200] [INSPIRE].

[2] S.S. Gubser, I.R. Klebanov and A.M. Polyakov, Gauge theory correlators from noncritical string theory, Phys. Lett. B 428 (1998) 105 [hep-th/9802109] [INSPIRE].

[3] E. Witten, Anti-de Sitter space and holography, Adv. Theor. Math. Phys. 2 (1998) 253 [hep-th/9802150] [INSPIRE].

[4] N. Beisert et al., Review of AdS/CFT Integrability: An Overview, Lett. Math. Phys. 99 (2012) 3 [arXiv:1012.3982] [INSPIRE].

[5] R.R. Metsaev and A.A. Tseytlin, Type IIB superstring action in $A d S_{5} \times S^{5}$ background, Nucl. Phys. B 533 (1998) 109 [hep-th/9805028] [INSPIRE].

[6] I. Bena, J. Polchinski and R. Roiban, Hidden symmetries of the $A d S_{5} \times S^{5}$ superstring, Phys. Rev. D 69 (2004) 046002 [hep-th/0305116] [INSPIRE]. 
[7] G. Arutyunov and S. Frolov, Foundations of the $A d S_{5} \times S^{5}$ Superstring. Part I, J. Phys. A 42 (2009) 254003 [arXiv:0901.4937] [INSPIRE].

[8] F. Delduc, M. Magro and B. Vicedo, An integrable deformation of the $A d S_{5} \times S^{5}$ superstring action, Phys. Rev. Lett. 112 (2014) 051601 [arXiv: 1309.5850] [INSPIRE].

[9] I. Kawaguchi, T. Matsumoto and K. Yoshida, Jordanian deformations of the $A d S_{5} \times S^{5}$ superstring, JHEP 04 (2014) 153 [arXiv:1401.4855] [INSPIRE].

[10] T.J. Hollowood, J.L. Miramontes and D.M. Schmidtt, An Integrable Deformation of the $A d S_{5} \times S^{5}$ Superstring, J. Phys. A 47 (2014) 495402 [arXiv:1409.1538] [INSPIRE].

[11] B. Vicedo, Deformed integrable $\sigma$-models, classical $R$-matrices and classical exchange algebra on Drinfel'd doubles, J. Phys. A 48 (2015) 355203 [arXiv:1504.06303] [INSPIRE].

[12] B. Hoare and A.A. Tseytlin, On integrable deformations of superstring $\sigma$-models related to $A d S_{n} \times S^{n}$ supercosets, Nucl. Phys. B 897 (2015) 448 [arXiv:1504.07213] [InSPIRE].

[13] K. Sfetsos, K. Siampos and D.C. Thompson, Generalised integrable $\lambda$ - and $\eta$-deformations and their relation, Nucl. Phys. B 899 (2015) 489 [arXiv: 1506.05784] [InSPIRE].

[14] C. Klimčík, $\eta$ and $\lambda$ deformations as E-models, Nucl. Phys. B 900 (2015) 259 [arXiv: 1508.05832] [INSPIRE].

[15] C. Klimčík and P. Ševera, Dual non-Abelian duality and the Drinfeld double, Phys. Lett. B 351 (1995) 455 [hep-th/9502122] [INSPIRE].

[16] C. Klimčík and P. Ševera, Poisson-Lie T duality and loop groups of Drinfeld doubles, Phys. Lett. B 372 (1996) 65 [hep-th/9512040] [INSPIRE].

[17] C. Klimčík, Yang-Baxter $\sigma$-models and dS/AdS T duality, JHEP 12 (2002) 051 [hep-th/0210095] [INSPIRE].

[18] C. Klimčík, On integrability of the Yang-Baxter $\sigma$-model, J. Math. Phys. 50 (2009) 043508 [arXiv:0802.3518] [INSPIRE].

[19] F. Delduc, M. Magro and B. Vicedo, On classical q-deformations of integrable $\sigma$-models, JHEP 11 (2013) 192 [arXiv:1308.3581] [INSPIRE].

[20] K. Sfetsos, Integrable interpolations: From exact CFTs to non-Abelian T-duals, Nucl. Phys. B 880 (2014) 225 [arXiv:1312.4560] [INSPIRE].

[21] T.J. Hollowood, J.L. Miramontes and D.M. Schmidtt, Integrable Deformations of Strings on Symmetric Spaces, JHEP 11 (2014) 009 [arXiv: 1407.2840] [INSPIRE].

[22] R. Borsato, Integrable strings for AdS/CFT, Ph.D. Thesis, Imperial College, London U.K. (2015) [arXiv: 1605.03173] [INSPIRE].

[23] T. Matsumoto and K. Yoshida, Integrable deformations of the $A d S_{5} \times S^{5}$ superstring and the classical Yang-Baxter equation - Towards the gravity/CYBE correspondence -, J. Phys. Conf. Ser. 563 (2014) 012020 [arXiv:1410.0575] [INSPIRE].

[24] D.C. Thompson, Generalised T-duality and Integrable Deformations, Fortsch. Phys. 64 (2016) 349 [arXiv:1512.04732] [InSPIRE].

[25] R. Borsato and L. Wulff, Target space supergeometry of $\eta$ and $\lambda$-deformed strings, JHEP 10 (2016) 045 [arXiv: 1608.03570] [INSPIRE].

[26] L. Wulff and A.A. Tseytlin, $\kappa$-symmetry of superstring $\sigma$-model and generalized $10 d$ supergravity equations, JHEP 06 (2016) 174 [arXiv: 1605.04884] [INSPIRE]. 
[27] A. Mikhailov, Cornering the unphysical vertex, JHEP 11 (2012) 082 [arXiv:1203.0677] [INSPIRE].

[28] G. Arutyunov, S. Frolov, B. Hoare, R. Roiban and A.A. Tseytlin, Scale invariance of the $\eta$-deformed $A d S_{5} \times S^{5}$ superstring, T-duality and modified type-II equations, Nucl. Phys. B 903 (2016) 262 [arXiv:1511.05795] [InSPIRE].

[29] R. Borsato, A.A. Tseytlin and L. Wulff, Supergravity background of $\lambda$-deformed model for $A d S_{2} \times S^{2}$ supercoset, Nucl. Phys. B 905 (2016) 264 [arXiv:1601.08192] [INSPIRE].

[30] Y. Chervonyi and O. Lunin, Supergravity background of the $\lambda$-deformed $A d S_{3} \times S^{3}$ supercoset, Nucl. Phys. B 910 (2016) 685 [arXiv:1606.00394] [INSPIRE].

[31] Y. Chervonyi and O. Lunin, Generalized $\lambda$-deformations of $A d S_{p} \times S^{p}$, Nucl. Phys. B 913 (2016) 912 [arXiv:1608.06641] [InSPIRE].

[32] K. Sfetsos and D.C. Thompson, Spacetimes for $\lambda$-deformations, JHEP 12 (2014) 164 [arXiv: 1410.1886] [INSPIRE].

[33] S. Demulder, K. Sfetsos and D.C. Thompson, Integrable $\lambda$-deformations: Squashing Coset CFTs and $A d S_{5} \times S^{5}$, JHEP 07 (2015) 019 [arXiv:1504.02781] [INSPIRE].

[34] G. Arutyunov, R. Borsato and S. Frolov, S-matrix for strings on $\eta$-deformed $A d S_{5} \times S^{5}$, JHEP 04 (2014) 002 [arXiv:1312.3542] [INSPIRE].

[35] G. Arutyunov, R. Borsato and S. Frolov, Puzzles of $\eta$-deformed $A d S_{5} \times S^{5}$, JHEP 12 (2015) 049 [arXiv: 1507.04239] [INSPIRE].

[36] H. Kyono and K. Yoshida, Supercoset construction of Yang-Baxter deformed $A d S_{5} \times S^{5}$ backgrounds, PTEP 2016 (2016) 083B03 [arXiv: 1605.02519] [INSPIRE].

[37] B. Hoare and S.J. van Tongeren, Non-split and split deformations of $\mathrm{AdS}_{5}$, J. Phys. A 49 (2016) 484003 [arXiv: 1605.03552] [InSPIRE].

[38] B. Hoare and S.J. van Tongeren, On Jordanian deformations of $A d S_{5}$ and supergravity, J. Phys. A 49 (2016) 434006 [arXiv: 1605.03554] [inSPIRE].

[39] D. Orlando, S. Reffert, J.-i. Sakamoto and K. Yoshida, Generalized type IIB supergravity equations and non-Abelian classical r-matrices, J. Phys. A 49 (2016) 445403 [arXiv: 1607.00795] [INSPIRE].

[40] B. Hoare and A.A. Tseytlin, Type IIB supergravity solution for the T-dual of the $\eta$-deformed $A d S_{5} \times S^{5}$ superstring, JHEP $10(2015) 060$ [arXiv:1508.01150] [INSPIRE].

[41] O. Hohm and H. Samtleben, Exceptional Form of D = 11 Supergravity, Phys. Rev. Lett. 111 (2013) 231601 [arXiv:1308.1673] [INSPIRE].

[42] O. Hohm and H. Samtleben, Exceptional Field Theory I: $E_{6(6)}$ covariant Form of M-theory and Type IIB, Phys. Rev. D 89 (2014) 066016 [arXiv:1312.0614] [InSPIRE].

[43] O. Hohm and H. Samtleben, Exceptional field theory. II. E $E_{7(7)}$, Phys. Rev. D 89 (2014) 066017 [arXiv:1312.4542] [INSPIRE].

[44] A. Baguet, O. Hohm and H. Samtleben, $E_{6(6)}$ exceptional field theory: Review and embedding of type IIB, PoS (CORFU2014) 133 [arXiv: 1506. 01065] [INSPIRE].

[45] Y. Sakatani, S. Uehara and K. Yoshida, Generalized gravity from modified DFT, arXiv: 1611.05856 [INSPIRE]. 
[46] W. Siegel, Superspace duality in low-energy superstrings, Phys. Rev. D 48 (1993) 2826 [hep-th/9305073] [INSPIRE].

[47] C. Hull and B. Zwiebach, Double field theory, JHEP 09 (2009) 099 [arXiv:0904.4664] [INSPIRE].

[48] O. Hohm, C. Hull and B. Zwiebach, Background independent action for double field theory, JHEP 07 (2010) 016 [arXiv: 1003.5027] [INSPIRE].

[49] O. Hohm, C. Hull and B. Zwiebach, Generalized metric formulation of double field theory, JHEP 08 (2010) 008 [arXiv: 1006.4823] [InSPIRE].

[50] E. Bergshoeff, T. de Wit, U. Gran, R. Linares and D. Roest, (Non-)Abelian gauged supergravities in nine-dimensions, JHEP 10 (2002) 061 [hep-th/0209205] [INSPIRE].

[51] J.J. Fernandez-Melgarejo, T. Ortín and E. Torrente-Lujan, The general gaugings of maximal $D=9$ supergravity, JHEP 10 (2011) 068 [arXiv:1106.1760] [INSPIRE].

[52] A. Le Diffon and H. Samtleben, Supergravities without an action: gauging the trombone, Nucl. Phys. B 811 (2009) 1 [arXiv:0809.5180] [inSPIRE].

[53] E. Musaev and H. Samtleben, Fermions and supersymmetry in $E_{6(6)}$ exceptional field theory, JHEP 03 (2015) 027 [arXiv:1412.7286] [INSPIRE].

[54] D.S. Berman and M.J. Perry, Generalized geometry and M-theory, JHEP 06 (2011) 074 [arXiv: 1008.1763] [INSPIRE].

[55] A. Coimbra, C. Strickland-Constable and D. Waldram, $E_{d(d)} \times \mathbb{R}^{+}$generalised geometry, connections and M-theory, JHEP 02 (2014) 054 [arXiv:1112.3989] [INSPIRE].

[56] O. Hohm and H. Samtleben, Consistent Kaluza-Klein truncations via exceptional field theory, JHEP 01 (2015) 131 [arXiv: 1410.8145] [INSPIRE].

[57] F. Ciceri, A. Guarino and G. Inverso, The exceptional story of massive IIA supergravity, JHEP 08 (2016) 154 [arXiv: 1604.08602] [INSPIRE].

[58] M. Graña and D. Marques, Gauged double field theory, JHEP 04 (2012) 020 [arXiv: 1201.2924] [INSPIRE].

[59] B. de Wit, H. Samtleben and M. Trigiante, The maximal $D=5$ supergravities, Nucl. Phys. B 716 (2005) 215 [hep-th/0412173] [INSPIRE].

[60] D.S. Berman, C.D.A. Blair, E. Malek and F.J. Rudolph, An action for F-theory: SL(2) $\mathbb{R}^{+}$ exceptional field theory, Class. Quant. Grav. 33 (2016) 195009 [arXiv:1512.06115] [INSPIRE].

[61] A. Baguet, O. Hohm and H. Samtleben, Consistent Type IIB Reductions to Maximal 5D Supergravity, Phys. Rev. D 92 (2015) 065004 [arXiv:1506.01385] [INSPIRE]. 